\title{
Intraoperative Navigation und computerassistierte Chirurgie in der MKG-Chirurgie
}

\author{
Onkologische Chirurgie und rekonstruktive Tumorchirurgie
}

\author{
Harald Essig, Martin Rücker, Frank Tavassol, Horst Kokemüller, Nils-Claudius Gellrich
}

\section{Zusammenfassung}

Die computerassistierte Planung ist ein grundlegender Bestandteil der Chirurgie der Tumoren im Kopf-HalsBereich. Nach multimodaler Bilddatenanalyse im Rahmen des TumorStagings können neben der Ausdehnung des Tumors auch die geforderten Resektionsgrenzen virtuell simuliert werden. Anhand dieser Information erfolgt die Rekonstruktionsplanung. Diese basiert meist auf virtuellen Spiegelungen der nicht betroffenen Seite und erlaubt 1. die Herstellung patientenspezifischer Implantate und 2., bei Verwendung der intraoperativen $\mathrm{Na}$ vigation, die Lage- und Formkontrolle der Rekonstruktion. Der Einsatz der Navigation bietet zudem die Möglichkeit, intraoperativ erhobene Befunde zu markieren und - als Novum - deren digitale Speicherung im weltweit akzeptierten DICOM-Format. Diese Dokumentation kann die interdisziplinäre Kommunikation zu Onkologen, Strahlentherapeuten und Radiologen verbessern. Neben der Resektionsund Rekonstruktionsplanung sowie dem intraoperativen Tumor-Mapping kann die computerassistierte Planung der Qualitätskontrolle dienen. Hierfür werden die präoperative virtuelle Planung mit der postoperativen Bildgebung fusioniert und die Abweichungen dargestellt.

\section{Intraoperative Navigation and Computer-Assisted Craniomaxillofacial Surgery}

Computer-assisted planning is an essential component of surgery for tumours in the head and neck region. Following analysis of multimodal imaging data in the course of tumour staging, not only the extent of the tumour but also the required resection margins can be simulated virtually. Planning of reconstruction procedures is then performed with the help of this information. This is based on a virtual reflection of the unaffected side and thus allows firstly the planning of patientspecific grafts and secondly, using intraoperative navigation, control of the position and shape of the reconstruction. The use of navigation also offers the possibility to record the intraoperative findings and, what is new, enables their digital storage in the worldwide accepted DICOM format. This documentation can help to improve the interdisciplinary communication between oncologists, specialists in radiation therapy, and radiologists. In addition to resection and reconstruction planning as well as intraoperative tumour mapping, computer-assisted planning can also serve for quality control. For this purpose the preoperative virtual planning data are fused with the postoperative imaging data in order to demonstrate any deviations.

\section{Einleitung}

Dreidimensionale (voxelbasierte) Datensätze gehören seit den 1980er-Jahren routinemäßig sowohl in die Behand-

OP-JOURNAL 2011; 27: 130-137

(c) Georg Thieme Verlag KG Stuttgart · New York DOI http://dx.doi.org/10.1055/s-0031-1280252 lungsplanung onkologischer Erkrankungen als auch in die Planung rekonstruktiver Maßnahmen nach ablativen tumorchirurgischen Eingriffen im Mund-, Kiefer- und Gesichtsbereich [1-5]. Hierbei ist das sog. Tumorstaging die Grundlage der operativen Therapie von Tumoren im Kopf-Hals-Bereich. Ein wesentlicher Bestandteil dieses Stagings ist die computerassistierte Analyse und Pla- nung. Dazu werden im Regelfall 3-dimensionale (3-D) Bilddaten unterschiedlicher Modalitäten, wie Computertomografie und Magnetresonanztomografie, erhoben. Die Bildanalyse wird idealerweise mit einer geeigneten Bildanalyseplattform durchgeführt. Diese erlaubt die Fusionierung der multimodalen Datensätze, die Planung der Resektion inkl. des geforderten Sicherheitsabstands, die Planung der Rekonstruktion, die Herstellung patientenspezifischer Implantate und erleichtert die interdisziplinäre Übermittlung selbst intraoperativ rekrutierter Daten (Tumormarkierung) z. B. an die Onkologie, Strahlentherapie, Pathologie und Radiologie. Im zeitlichen Verlauf können im Rahmen des Re-Stagings weitere Bilddaten fusioniert werden.

Im Gegensatz zu neurochirurgischen Anwendungsgebieten, dem Ursprung der computerassistierten Planung und intraoperativen Navigation, werden in den Disziplinen der Kopf-Hals-Chirurgie keine Bewegungspfade zum Auffinden vorhandener Raumforderungen geplant, sondern Deformationen jedweder Genese (angeboren, erworben) analysiert, 3-dimensional Sicherheitsabstände simuliert und Rekonstruktionen virtuell geplant $[2-4,6,7]$. Die Vorlage (Template) für die Rekonstruktion stellt dabei meist die Spiegelung der nicht betroffenen kontralateralen Seite dar.

Der Einsatz der computerassistierten Planung in der MKG-Chirurgie wird im Folgenden exemplarisch dargestellt.

\section{Computerassistierte Planung in der Tumorchirurgie}

Die 3-dimensionale Bildgebung bildet die Basis für die multiplanare und 3-dimensionale Analyse im Rahmen der computerassistierten Planung. Dabei können Bilddaten mehrerer unterschiedlicher Modalitäten wie Computertomografie (CT) und Magnetresonanztomografie (MRT) fusioniert und die Abbildungsvor- 

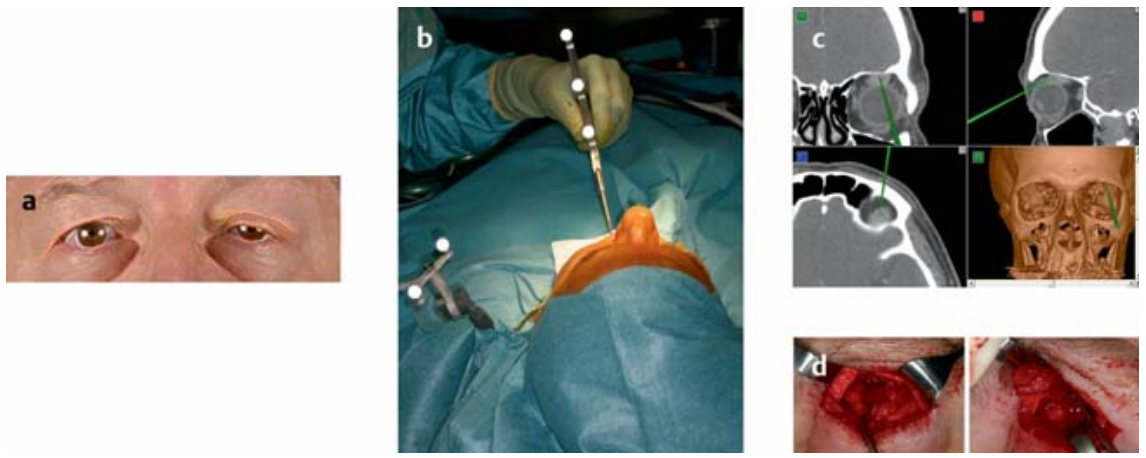

Abb. 1 a bis d Biopsie einer intraorbitalen, extrakonalen Raumforderung. Klinisches Bild (a), Anwendung der intraoperativen Navigation (b), Visualisierung in Echtzeit (c), Zugangsweg über temporäre Marginotomie am superomedialen Orbitarand (d).
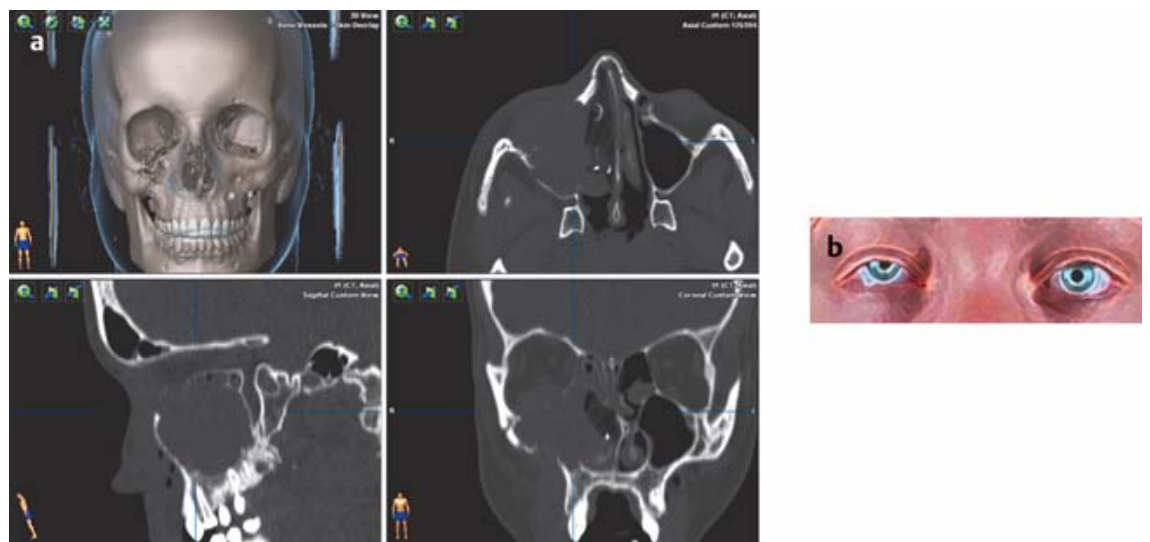

Abb. 2 a und $\mathbf{b}$ Initiale CT-Diagnostik einer Raumforderung in der rechten Kieferhöhle. Multiplanare CT-Analyse (a), klinisches Bild (b).

teile der jeweiligen Bilddaten kombiniert dargestellt werden. Auf diese Weise können z.B. Tumorausdehnung (vorzugsweise MRT-Bildgebung) und knöcherne Beteiligung (CT-Bildgebung) virtuell markiert und visualisiert werden. Diese Markierung (Segmentierung) kann durch den geforderten Sicherheitsabstand virtuell augmentiert werden. Daraus können sich nicht nur Konsequenzen für den ablativen Eingriff ergeben, sondern auch für das Ausmaß der Rekonstruktion und die Auswahl einer geeigneten Rekonstruktionsmethode.

Moderne Bildanalyseplattformen erlauben neben der multimodalen Bildanalyse die Segmentierung von Tumoren, Erweiterung dieser Segmentierung um einen definierten Sicherheitsabstand, die Simulation der knöchernen Rekonstruktion, den Import von präformierten 3-dimensionalen Rekonstrukten wie Titangitterstrukturen sowie die Bildfusion der Planung mit dem postoperativen Ergebnis.

\section{Computerassistierte Planung und intraoperative Navigation in der Tumorchirurgie}

Die intraoperative Anwendung der präoperativen Planung wird mit der intraoperativen Navigation realisiert. Hierbei sind Arbeitsschritte für die Referenzierung (Fusion des Bilddatensatzes mit dem Patienten) und Registrierung der räumlichen Lage notwendig (vgl. vorhergehender Artikel Wilde/Schramm/Allgemeines). Die Referenzierung kann über Landmarken erfolgen. Dazu werden eindeutig identifizierbare Strukturen, die über den geplanten chirurgischen Zugangsweg erreichbar sind, verwendet. Typischerweise werden dazu Zahnmerkmale oder anatomische Foramina gewählt. Die Genauigkeit lässt sich jedoch durch nicht invasive oder invasive Referenzierungsmethoden verbessern. Für die klinische Routine haben sich individuell laborgefertigte Tiefziehschienen für den Oberkiefer sowie die invasive Reben (Synthes Kreuzschlitzschraube 2.0, Synthes, Umkirch) bei unbezahntem ferenzierung mittels Titanminischrau- oder mobilem Oberkiefer bewährt. In der rekonstruktiven Tumorchirurgie ist die Insertion von Titanschrauben deutlich außerhalb der potenziellen Tumorresektionsgrenzen zu favorisieren.

Die Registrierung erfolgt über einen Reflektorstern, der entweder im Bereich der Schädelkalotte oder an einer metallischen Kopfhaltevorrichtung (MayfieldKlemme) fixiert wird.

Die virtuelle Planung erleichtert nicht nur die präoperative Analyse, sondern wird v.a. für die virtuelle Rekonstruktion (true-to-original) eingesetzt. Diese kann einerseits für die Herstellung patientenspezifischer Implantate verwendet werden (s.u.), andererseits jedoch, gemeinsam mit einem Ortungssystem (Navigation), unmittelbar den operativen Eingriff begleiten. Dies erleichtert die Tumorresektion durch die Unterstützung der anatomischen Orientierung, v.a. in schädelbasisnahen Bereichen, und durch die Visualisierung der 3-dimensionalen Sicherheitszone. Wichtig ist zudem das Einsegmentieren von wichtigen anatomischen Strukturen, wie z.B. der A. carotis interna oder des Sehnervs. Intraoperative Informationen, wie Entnahmestellen von Randschnitten und makroskopisch imponierende, nicht resektable Tumoranteile, können durch den Chirurgen im 3-D-Datensatz markiert und gespeichert werden. Die digitale Weitergabe dieser Informationen kann dann in der Bestrahlungsplanung berücksichtigt werden oder ergänzt die räumliche Orientierung und Zuordnung im Rahmen der pathologisch-anatomischen Begutachtung.

\section{Biopsien}

Raumforderungen, die sich unverschieblich an knöchernen Strukturen befinden, lassen sich mithilfe der intraoperativen Navigation auffinden. Das ist umso entscheidender, je näher die räumliche Beziehung zu wichtigen Nachbarstrukturen lokalisiert ist (z.B. N. opticus) und je unübersichtlicher sich der Zugangsweg darstellt (z.B. posteriorer Bereich der knöchernen Orbitawände). Hilfreich ist die Navigation jedoch auch dann, wenn sich die Raumforderung im Weichgewebe unmittelbar benachbart zu knöchernen (immobilen) Strukturen befindet und anatomische Nachbarstrukturen gefährdet sind. 


\section{Fallbeispiel 1}

Eine unklare intraorbitale, extrakonale Raumforderung (Abb.1), nicht abzugrenzen vom M. rectus superior und bis zum N. opticus reichend, wurde zunächst 3-dimensional analysiert und navigationsgestützt lokalisiert. Mit einer vom Navigationssystem erfassten Biopsiezange ließ sich dann gezielt Gewebe für die histologische Begutachtung resezieren. Es zeigte sich ein schlecht differenziertes Karzinom, welches mit einer Metastase eines Nierenzellkarzinoms vereinbar war.

Die computerassistierte Planung der Rekonstruktion kann für die Herstellung patientenspezifischer Implantate verwendet werden.

\section{Resektionen}

Für die Planung der computerassistierten Resektion eignen sich v.a. Prozesse, die sich im Bereich der nicht beweglichen Knochen des Gesichtsschädels bzw. Hirnschädels befinden, d.h. v.a. schädelbasisnahe Tumoren und Oberkiefertumoren. Ein Ziel, neben der Rekonstruktionsplanung, ist die Analyse der Resektionsgrenzen und der Operabilität. Wichtig hervorzuheben ist, dass die 3-dimensionale Markierung von Tumorvolumina eine rein makroskopische und volumendatensatzbasierte Maßnahme ist, die keine sichere Information über Gewebeverschieblichkeit, Elastizität oder Infiltrationen erlaubt; ebenso ist das Einsegmentieren eines Sicherheitsabstands vergleichbar der klinischen Entscheidung einer Festlegung eines 3-dimensionalen Sicherheitsabstands, d.h. es dient der Realisierung einer vollständigen Resektion, ohne dass man damit aber die histopathologischen Tumorgrenzen sicher erfassen könnte. Die multimodale Bildanalyse erlaubt Aussagen zu Ausdehnung sowie etwaigen Infiltrationen und Ummauerung von hirnversorgenden Gefäßen und ist Grundlage einer interdisziplinären Tumorkonferenz.

\section{Fallbeispiel 2}

Eine 19-jährige Patientin stellte sich mit einer rechtsseitigen Wangenschwellung, einem Bulbushochstand und Nasenbluten vor (Abb. 2). In der Diagnostik zeigte sich ein sinunasales Karzinom der rechten Kieferhöhle (pT4NOMO). Initial wurden Computertomografie- und Magnetresonanztomografiedatensätze fusioniert
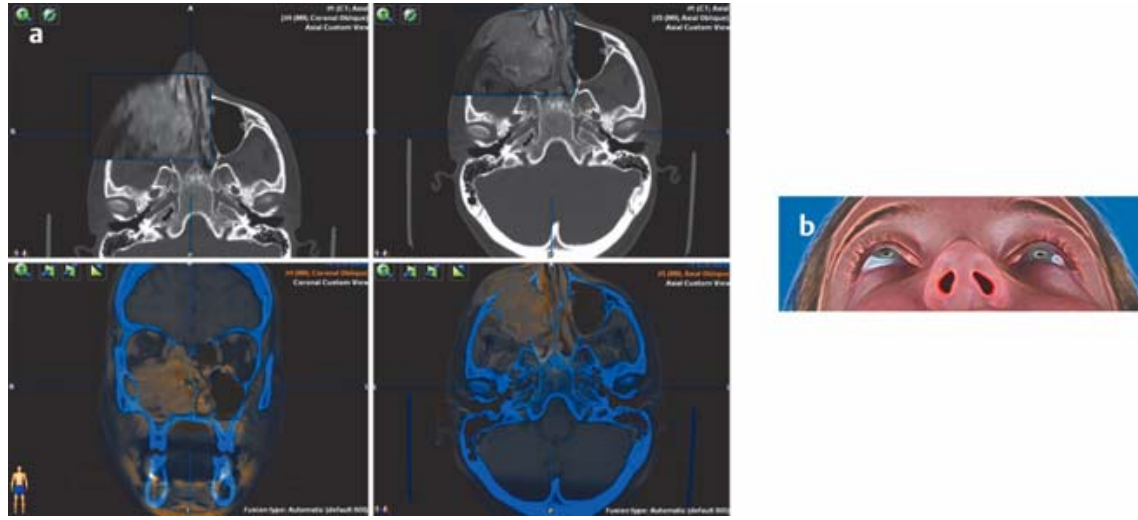

Abb. 3 a und b Fusionierung mehrerer Bildmodalitäten im Rahmen der 3-D-Analyse. Computertomografie (blau) mit der Magnetresonanztomografie (gelb) (a). Klinisches Bild aus der Froschperspektive mit deutlicher Schwellung der rechten Wange (b).
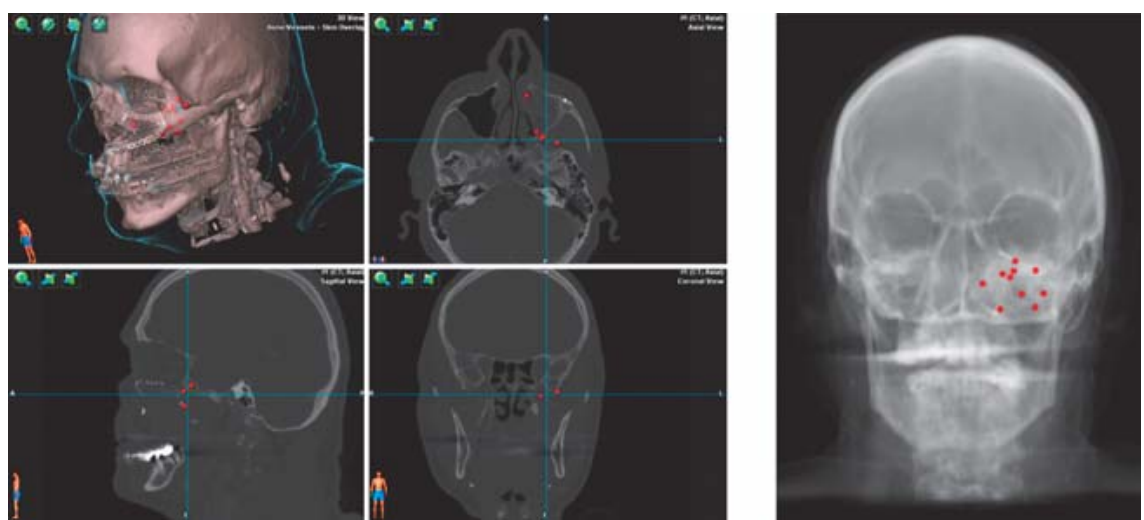

Abb. 4 Markierung der Lokalisation von intraoperativen Gewebeentnahmen in Form von digitalen Voxel (rote Punkte).
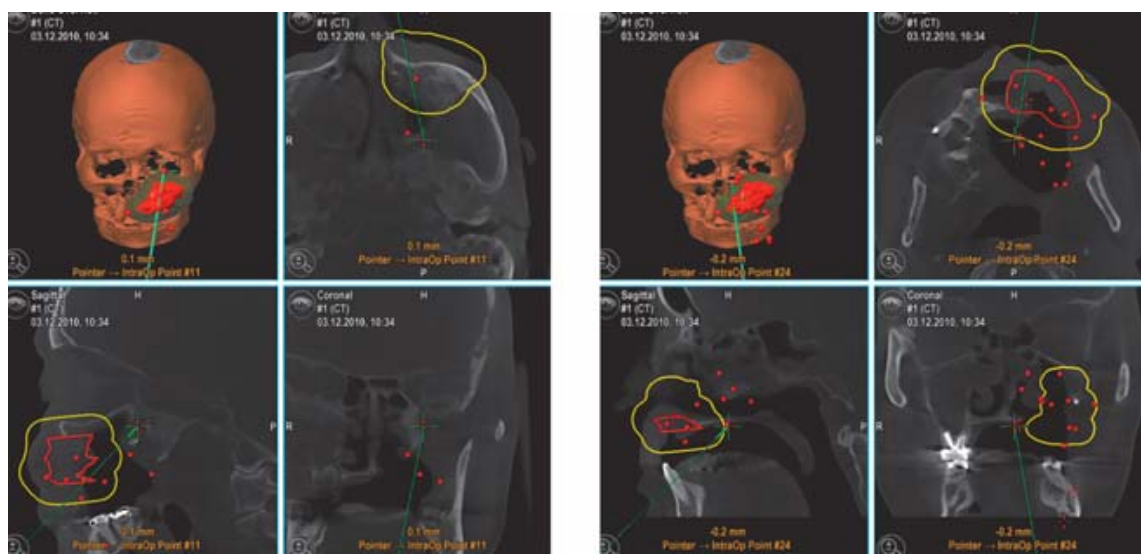

Abb. 5 Intraoperatives Markieren der Schnellschnittlokalisationen. Tumormasse im MRT (rot umrandet), virtuelle Sicherheitszone (gelb umrandet).

(Abb. 3), um die knöcherne Beteiligung und die Tumorausdehnung zu analysieren. Dazu erfolgten die Segmentierung des radiologisch sichtbaren Tumors in der MRT-Bildgebung und die virtuelle Simulation der Resektionsgröße durch Einblenden des virtuellen Sicherheitsabstands.

\section{Tumor-Mapping}

Ein typisches Problem bei der Tumorresektion (ohne Einsatz der Navigation) stellt die korrekte Benennung und $\mathrm{Zu}$ ordnung der Randschnitte dar. Fehlerquellen sind v.a. die unzureichenden anatomischen Angaben zu einer Proben- 


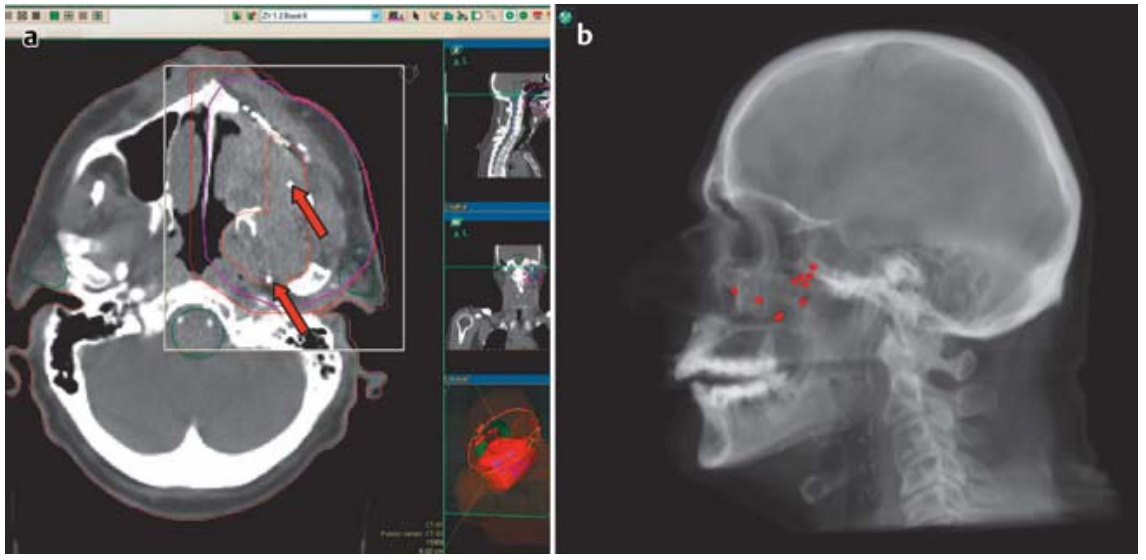

Abb. $\mathbf{6}$ a und $\mathbf{b}$ Weitergabe der Informationen an die Strahlentherapie. Bestrahlungsplanung (a) mit tumorpositiven Markierungen (Pfeile) und virtuelles FRS zur Orientierung (b).
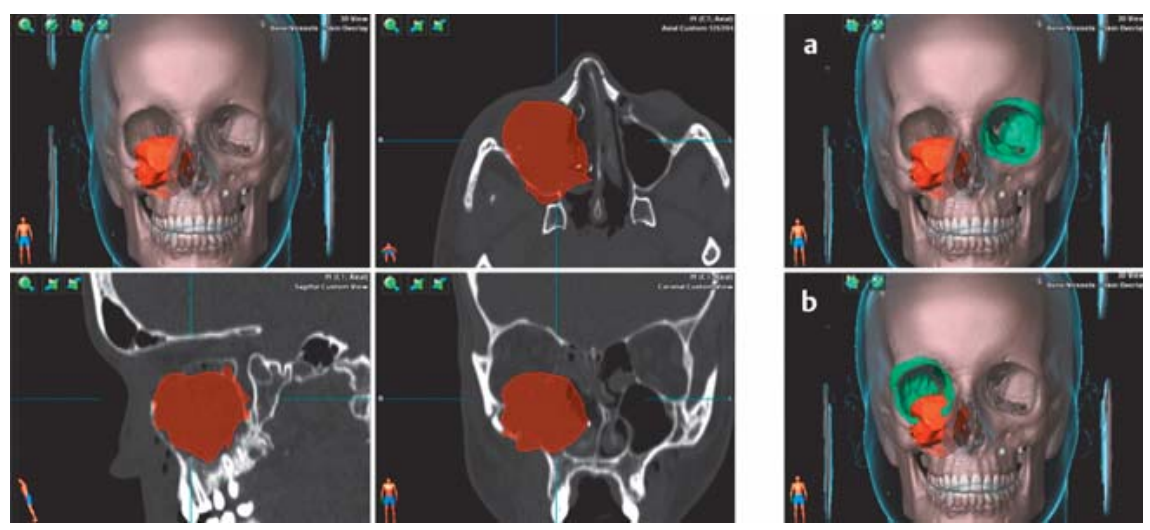

Abb. $7 \mathbf{a}$ und $\mathbf{b}$ Rekonstruktionsplanung nach Segmentierung von Tumor und gesunder Orbita (a). Spiegelung der gesunden Orbita zur betroffenen Seite (b).
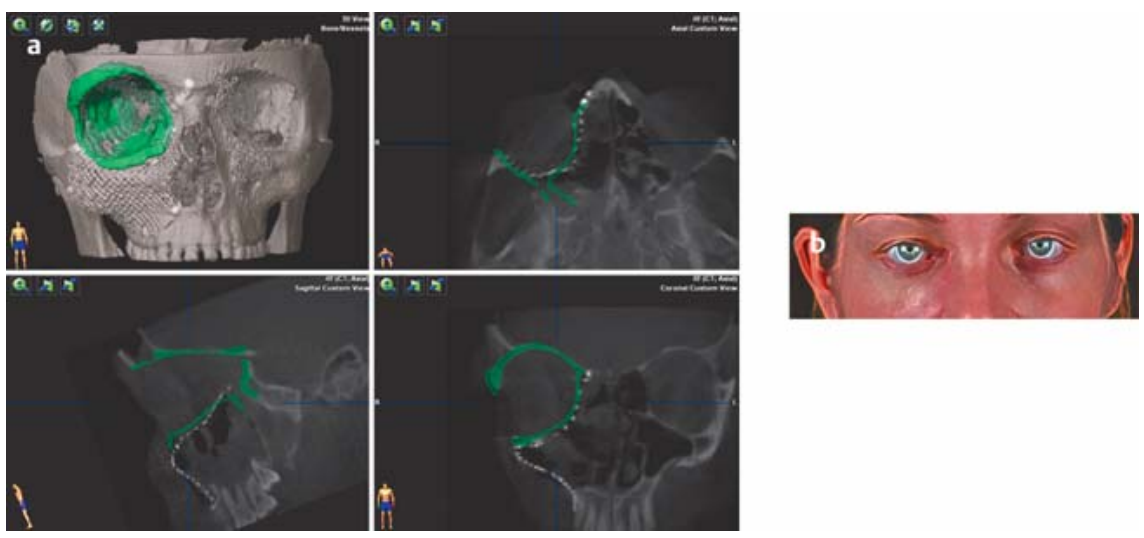

Abb. 8 a und $\mathbf{b}$ Fusion der präoperativen Planung (grün) mit der postoperativen 3-D-Bildgebung (a). Fallbeispiel 2 - klinisches Bild der Patientin 2 Wochen postoperativ (b).

entnahme und die richtige Zuordnung der Probe zur Benennung nach mündlichem Diktat des Operateurs. Die intraoperative Navigation erlaubt nicht nur die Verwendung virtueller Resektionsplanungen, sondern auch die virtuelle Markierung dieser Randschnitte für die eindeutige anatomische Lokalisation im Koordinatensystem und die eindeutige
Benennung. Mit diesen zusätzlichen Informationen, die im DICOM-Format abgespeichert werden können, ist die postoperative Zuordnung der histologischen Ergebnisse zuverlässig und deutlich vereinfacht (Abb.4). Diese postoperative Zuordnung kann z.B. auch im Langzeitverlauf einer Tumorbehandlung von Bedeutung sein, d.h. wenn im Rahmen des
Re-Stagings erneute 3-D-Datensätze erstellt werden, so könnten diese mit vorangegangenen Datensätzen fusioniert und auch im Hinblick auf die „gemappten" vormals vorgenommenen Markierungen verglichen werden.

Das genannte Navigationssetting erlaubt zudem auch die digitale Speicherung intraoperativer Befunde in Echtzeit (Abb.5). Diese Informationen können z. B. die Lage von Tumoranteilen, die makroskopisch für den Operateur erkennbar sind, jedoch aus Gründen der Lagebeziehung zu lebenswichtigen Strukturen nicht resektabel sind (R2-Situation), dokumentieren und werden postoperativ dem Strahlentherapeuten übermittelt. Dieser kann den mit intraoperativen Informationen angereicherten Datensatz importieren und für die Bestrahlungsplanung berücksichtigen (Abb. 6).

Das Navigationssetting erlaubt die digitale Speicherung intraoperativer Befunde und ermöglicht die digitale und verlustfreie Weitergabe dieser Befunde an Strahlentherapeuten, Radiologen und Onkologen.

\section{Rekonstruktionen}

Die Analyse der Tumor- und Resektionsgrenzen zeigt den Umfang der notwendigen Rekonstruktionsmaßnahmen. Kombiniert knöcherne und weichgewebige Defekte werden meist mit osteokutanen Transplantaten oder mit alloplastischem Material wie Titangitterstrukturen und faziokutanen Transplantaten rekonstruiert [8]. Bei Mittelgesichtsrekonstruktionen mit Beteiligung der knöchernen Orbita und fazialen Kieferhöhlenwand hat sich der Einsatz von Titanmeshimplantaten bewährt. Diese können passgenau geformt werden und erlauben die knöcherne True-to-Original-Rekonstruktion v.a. filigraner Strukturen des Mittelgesichts.

Bei der Verwendung solcher patientenspezifischer, alloplastischer Implantate gelten dieselben Grundregeln wie beim avaskulären Knochentransplantat, d.h. nur der sichere 3-dimensionale vaskularisierte Gewebemantel (externes und internes Lining) stellt auch die biologischadäquate Rekonstruktion sicher, sodass z.B. auch eine spätere Strahlentherapie ohne Exposition des Meshs möglich ist.

Bei unilateralem Tumorwachstum kann die virtuelle Spiegelung der kontralateralen Seite als Anhalt für die Rekonstruk- 
tion dienen. Diese virtuelle Rekonstruktionsplanung lässt sich exportieren. Die Herstellung eines Stereolithografiemodells bietet die Möglichkeit, Titanmeshimplantate präoperativ zu formen (vgl. vorhergehender Artikel Wilde/Schramm/ Sekundärrekonstruktionen, Abb. 7). Dieser Schritt ermöglicht eine präzise und zuverlässige Rekonstruktion.

\section{Fallbeispiel 2 - Fortsetzung}

Bei der o.g. Patientin zeigte sich u.a., dass der rechte Orbitaboden durch das Tumorwachstum nach kranial verdrängt und arrodiert war. Intraoperativ ergaben die Randschnittkontrollen zum intraorbitalen Weichgewebe Tumorfreiheit, sodass die Entscheidung auf eine Bulbusund Orbita- und damit funktionserhaltende Tumorresektion gefällt wurde. Für einen langfristigen Funktionserhalt war jedoch eine originalgetreue Rekonstruktion der Orbita erforderlich, wobei die gespiegelte intakte kontralaterale Seite als virtuelle Vorlage diente. Die virtuelle Schablone („Blaupause“) von der nicht betroffenen Gegenseite ermöglichte die infrarotbasierte Navigation und damit die „true-to-original“--Rekonstruktion, um eine Fehlstellung des Bulbus und Doppelbildwahrnehmung zu vermeiden (Abb. 7 und 8).

\section{Qualitätskontrolle}

Eine postoperative 3-dimensionale Bildgebung erfolgt in der Regel zur Dokumentation der Tumorresektion (für Tumornachsorge und ggf. Re-Staging) und findet, sofern indiziert, in der Bestrahlungsplanung Verwendung. Für die Qualitätskontrolle der Rekonstruktion kann dieser Bilddatensatz in die Bildanalyseplattform importiert und mit der virtuellen Rekonstruktionsplanung fusioniert werden (Abb. 8).

\section{Prothetisch orientiertes „backwards planning“ zur adäquaten knöchernen Kieferrekonstruktion}

Die virtuelle Operationsplanung (computerassistierte präoperative Planung; CAPP) ist die Grundlage für die intraoperative Navigation und für die Herstellung patientenspezifischer Implantate. Nach ausgedehnten Resektionen des Unterund/oder Oberkiefers werden die Rekonstruktionen 1- oder 2-zeitig durchgeführt. Je nach den Gewebeverhältnissen der Empfängerregion (Stichwort: „ersatzschwaches Lager") kommen avaskuläre oder vaskularisierte Knochen-
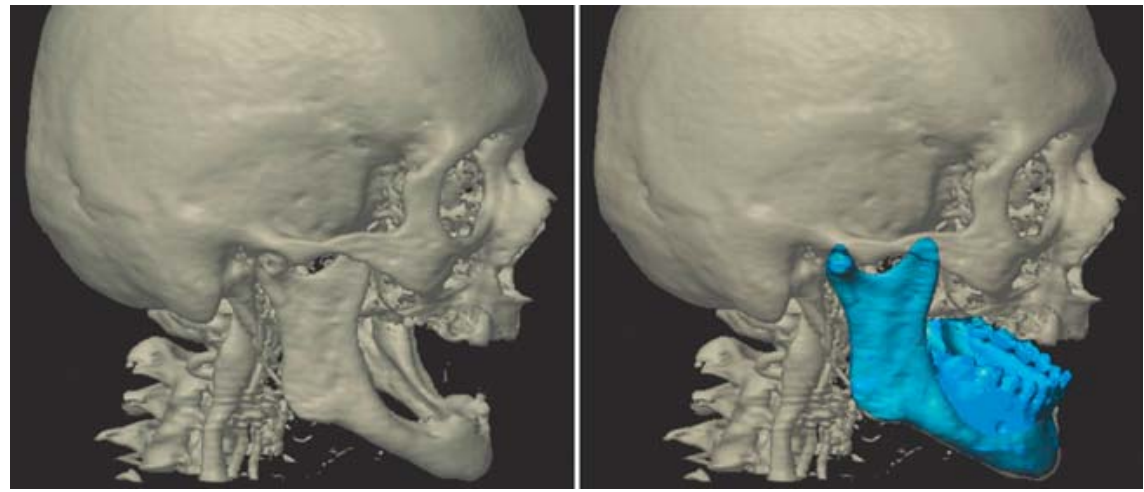

Abb.9 Initialer CT-Datensatz vor ablativer Tumorchirurgie (Hemimandibulektomie rechts). Virtuelle Rekonstruktion des Unterkiefers einschließlich Zahnersatz in blau (iPlan, Brainlab).
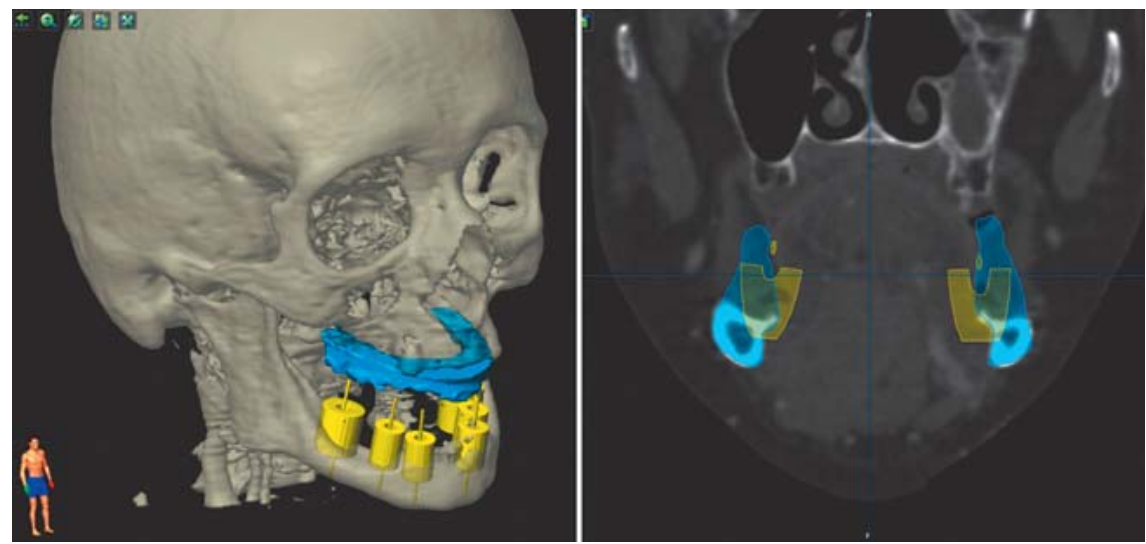

Abb. 10 Import eines idealen Oberkiefermodells (blau), angestrebte Position für enossale Zahnimplantate (gelbe Achse) mit dem dazu notwendigen Knochenangebot (gelber Hohlzylinder).
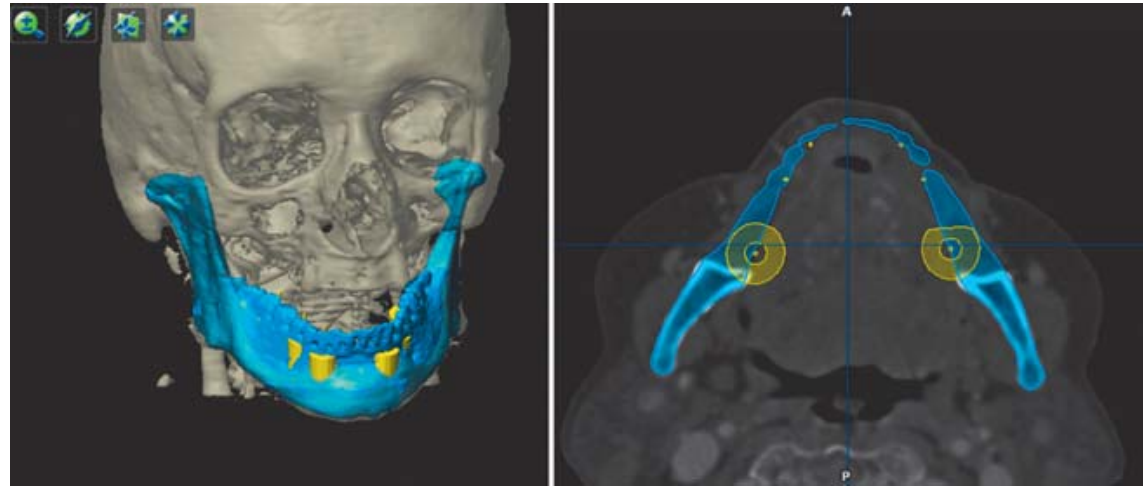

Abb. 11 Diskrepanz zwischen einer an der Außenkontur orientierten Unterkieferrekonstruktion (blau) und dem für Zahnimplantat-insertionen gewünschten Knochenangebot (ideale Implantatposition, gelber Hohlzylinder).

transplantate für die Rekonstruktion zur Anwendung. Traditionell wurden knöcherne Rekonstruktionen oder alloplastische Überbrückungsplatten in Entsprechung zur Außenkontur des originalen Unterkiefers geplant (Abb.9). Simultan oder im Rahmen einer Zweitoperation wurden dann vaskularisierte oder avaskuläre Knochentransplantate an die late- ral befindlichen Rekonstruktionsplatten fixiert.

Bisher wenig Beachtung fand dabei eine zahnärztlich-prothetisch orientierte Rückwärtsplanung: das grundsätzliche Ziel einer knöchernen Wiederherstellung der Kiefer ist nicht nur in der Wiederherstellung einer Außenkontur eines Gesichts zu sehen, sondern ganz 

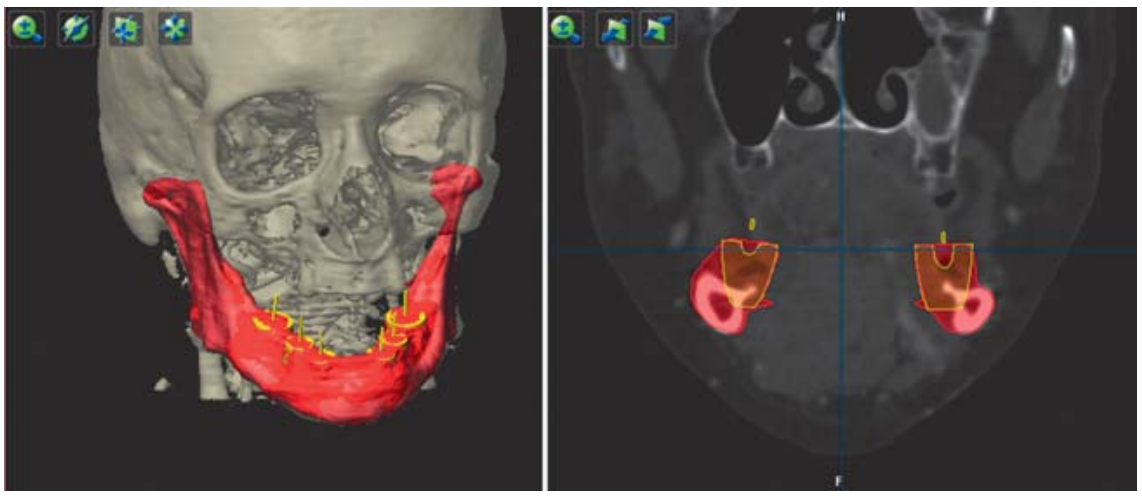

Abb. 12 Virtuelles Modellieren eines zur Implantatinsertion geeigneten Unterkiefers (Funktion SmartShaper, iPlan, Brainlab).
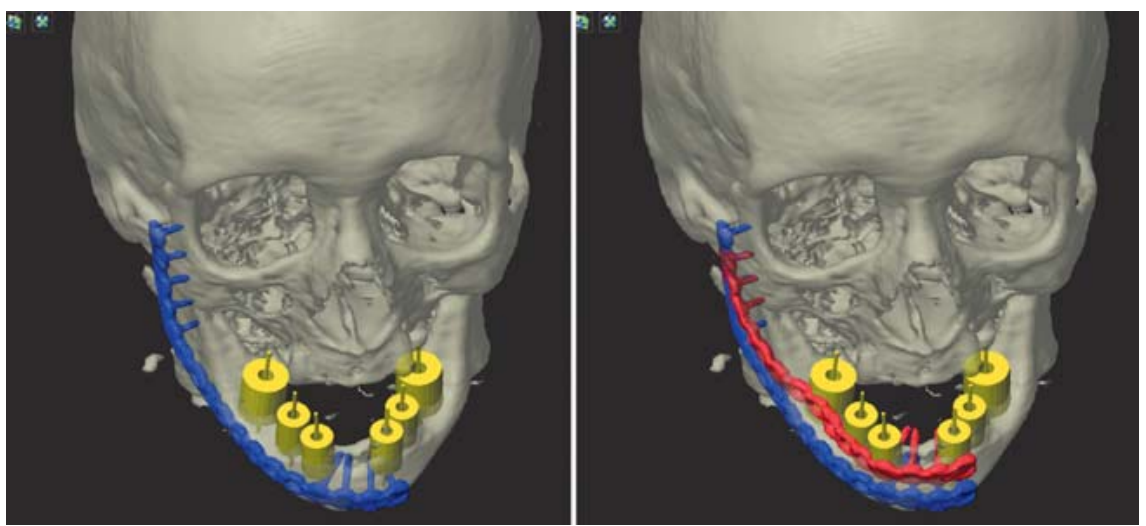

Abb.13 Herstellen einer patientenspezifischen Überbrückungsplatte (rot) im Vergleich zur einer lateral an der Außenkontur orientierten Plattenposition (blau).

wesentlich in der potenziellen Aufnahmemöglichkeit von enossalen Zahnimplantaten, auf denen dann ein implantatgetragener Zahnersatz verankert werden kann (Abb. 10).

Hierzu ist unabdingbar, dass die Relation von Unter- und Oberkiefer zueinander, ebenso wie die Zahnachsenstellung, Berücksichtigung findet. Ausgehend davon kann die Zahnimplantatposition virtuell geplant werden (Abb. 11 und 12), wobei immer ein definiertes Minimum von vaskularisiertem Knochen um den jeweiligen Implantatzylinder vorhanden sein muss (mind. 2-3 mm). Aus diesen Dimensionsvorgaben ergeben sich dann in der Rückwärtsplanung die Form, das Volumen und die Position eines notwendigen Knochentransplantats und letztlich die Innen- bzw. Außenkontur der patientenspezifischen Überbrückungsplatte (Abb. 13).

Diese Betrachtungsweise fand früher nicht hinreichend Beachtung, sodass insbesondere die transversale Breite eines späteren Knochentransplantats nicht den individuellen Anforderungen ent- sprach. Daraus resultierten Unterkieferrekonstruktionen, die oft deutlich zu weit lateral angeordnet waren, um problemlos als Grundlage für eine spätere orale Rehabilitation mit dentalen Implantaten geeignet zu sein (Abb. 11). Zusätzlich zu der ungünstigen lateralen Positionierung der Knochentransplantate war auch die Vertikalposition in der Regel inadäquat. Mithilfe der Rückwärtsplanung („backwards planning“) über die Autosegmentierung der Bildanalyseplattform (iPlan 3.0, BrainLAB Feldkirchen) kann die ideale dentale Implantatposition bereits vor der knöchernen Rekonstruktion geplant werden (Abb. 10). Die geringe transversale Breite der üblichen Knochentransplantate wird durch eine weiter lingualwärts orientierte Position ausgeglichen. Diese vorteilhafte Lokalisation wird durch die virtuell geplante und später geformte patientenspezifische Rekonstruktionsplatte intraoperativ vorgegeben.

Der hier aufgezeigte Planungsablauf ist wichtiger Bestandteil eines automatisierten digitalen Workflows. Aus der durch Autosegmentierung - und damit
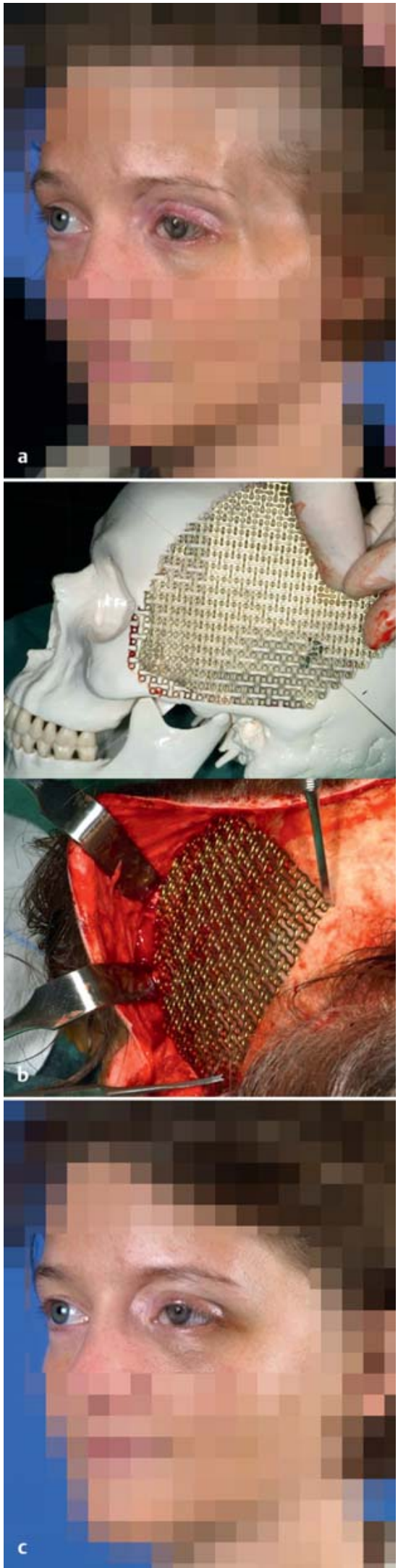

Abb. 14a bis c Korrektur eines „temporal hollowing" bei Anophthalmus links. Das Konturdefizit im Schläfenbereich links ist nach Rotation eines M.-temporalis-Lappens zur Auskleidung der linken Orbita entstanden, prä(a), intra- (b) und postoperativ (c) 


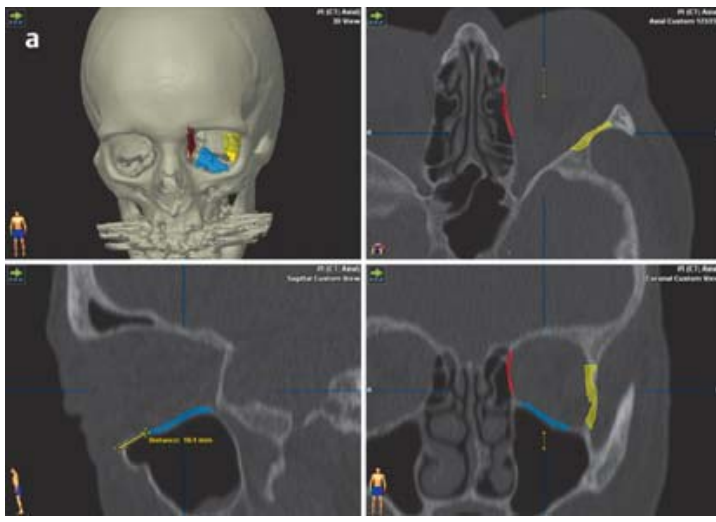

Abb. 15a und b Endokrine Orbitopathie (mit Betonung der linken Seite), virtuelle Planung (a), klinische Situation (b).
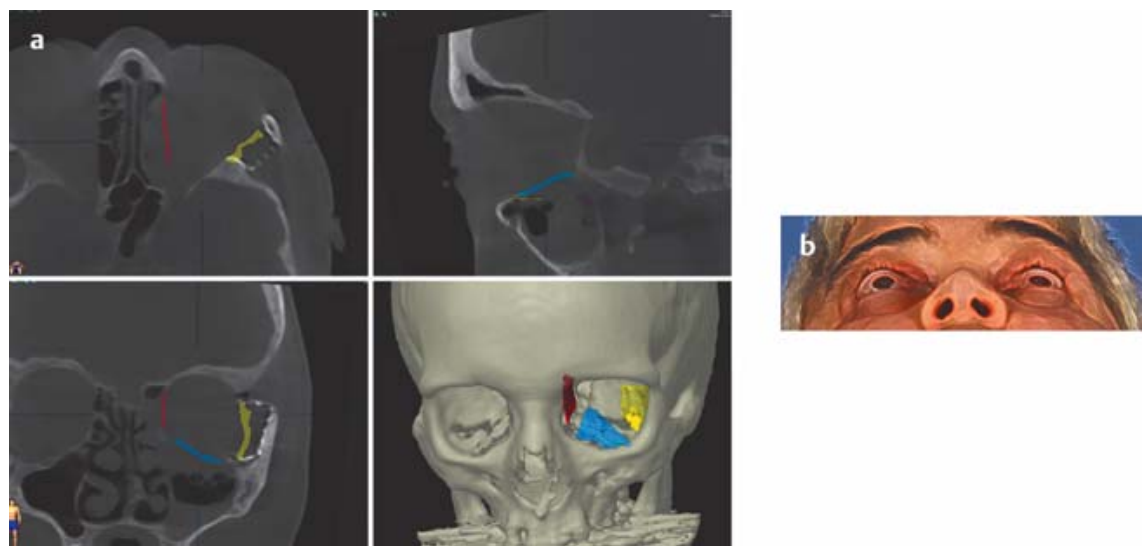

Abb. 16a und b Fusion der präoperativen virtuellen Planung mit der postoperativen Bildgebung (digitale Volumentomografie) in multiplanarer Ansicht. Beachte: Konturen des 3-D-Titangitterkäfigs in der Temporalgrube (a), klinische Situation (b).

unabhängig von zahnärztlichem Abdruck, Modell und Kiefermodell - entstandenen Planung wird ein patientenspezifisches virtuelles Modell erstellt, anhand dessen die Idealform einer knöchernen Unterkieferrekonstruktion ebenso perfekt geplant werden kann wie die dazugehörige patientenspezifische alloplastische Überbrückungsplatte. Eine gelungene oromandibuläre Rehabilitation mit aufeinander abgestimmten autogen/alloplastischen Rekonstruktionsmaßnahmen bedeutet nach Verlust von Kieferabschnitten im Ober- und/oder Unterkiefer einen Zugewinn an Lebensqualität und eine Perspektivenerweiterung für die betroffenen Tumorpatienten [9].

Die knöcherne Wiederherstellung eines Kiefers ist unter dem Gesichtspunkt der oralen Rehabilitation mit dentalen Implantaten zu sehen.

\section{Rekonturierung von Hirn- und/oder Gesichtschädelkonturdefekten}

Eine unmittelbare oder mittelbare Folge von onkologischen Eingriffen im Bereich des Hirn- oder Gesichtsschädels stellt das Temporal-Hollowing dar (Abb. 14). Hierbei handelt es sich in der Regel um einen Volumenverlust aufgrund einer Atrophie des M. temporalis nach Denervation oder Devaskularisierung. Diese Kontureinsenkung liegt zumeist im sichtbaren Bereich und kann für die Patienten extrem störende Auswirkungen haben. Sowohl beim ein- als auch beim beidseitigen Temporal-Hollowing besteht oft ein Handlungsbedarf, um die Lebensqualität der Patienten zu verbesssern. Klassische Methoden der Rekonstruktion zielen hier manchmal lediglich auf die Wiederherstellung der knöchernen Temporalschuppe ab. Adäquate Methoden sind sowohl auf das fehlende Volumen von Knochen als auch Weichgeweben ausgerichtet. Neben autogenen Rekonstruktionsverfahren (Fett, Kno- chen) bieten sich heute auch patientenspezifische Implantate an, die eine präzise Wiederherstellung der Außenkonturen vom lateralen Orbitrand und Jochbogen bis in den präaurikulären Bereich gestatten. Dazu hat sich auch der Einsatz von $0,6 \mathrm{~mm}$ dicken 3-D-Meshes (Synthes, Umkirch) bewährt.

Hier können komplexe 3-D-Gitterstrukturen dergestalt patientenspezifisch vorgeformt werden, dass sie für einen exakten Konturausgleich von fehlendem Hart- und/oder Weichgewebe sorgen.

Wurden in der Vorzeit noch Füllsubstanzen für den evtl. Hohlraum zwischen Mesh und der Gewebeoberfläche in der Tiefe eingesetzt, so wird heute auf deren Einsatz komplett verzichtet. Diese Rekonstruktionsmethode hat sich als effizient und adäquat erwiesen und bei mehr als 100 Rekonstruktionen keine Komplikationen durch Dehiszenzen oder Inkompatibilitäten erbracht.

\section{Computerassistenz für die chirurgi- sche Behandlungsplanung und Rekon- struktion bei Patienten mit therapie- resistenter endokriner Orbitopathie}

Zwar handelt sich bei der endokrinen Orbitopathie nicht um eine Tumorerkrankung, dennoch stellen die Volumenvermehrungen im Sinne einer intraorbitalen Raumforderung und die damit einhergehenden Funktionseinschränkungen für die Orbita eine besondere Herausforderung an den Chirurgen. Ein operatives Vorgehen kommt in der Regel dann zum Einsatz, wenn eine Medikamenten- und/oder Einsatz von Strahlentherapie nicht zum gewünschten Erfolg geführt haben.

Die nachfolgenden Abbildungen zeigen die Computerassistenz in der präoperativen Behandlungsplanung, bei intraoperativer navigationsgestützter Resektion von Orbitawandabschnitten und bei der Rekonstruktion durch patientenspezifische 3-D-Titangitterstrukturen, die wie hier als innovatives Verfahren gezeigt - den prolabierenden Weichgewebeinhalt aus der Temporalgrube fernhalten, ohne dass diese Distanzhalter den Patienten im Bereich der äußeren Schläfenkonturen kosmetisch beeinträchtigen würden. Das wahrscheinlich auch durch kein anderes chirurgisches Verfahren komplett zu lösende Problem von Doppelbildern nach einer chirurgischen Orbitawanddekompression kann durch die vorgezeichnete Methode minimiert wer- 
den (Abb. 15). Grundlage dafür sind folgende Erkenntnisse, die sich erst ausschließlich in der Auseinandersetzung mit der 3-dimensionalen Behandlungsplanung dieser Pathologien ergeben haben.

- Der Erhalt des Orbitabodens über eine Distanz von $0,8-1,0 \mathrm{~cm}$, gemessen vom Infraorbitalrand nach posterior, reicht für die Stabilisierung einer Vertikalposition des Bulbus aus. Die dahinter gelegenen Wandanteile des Orbitabodens können ohne Auswirkung auf die vertikale Position des Bulbus sorgfältig entfernt werden.

- Für die Sicherung der transversalen Position des Orbitainhalts ist das Belassen der Übergangszone zwischen Orbitaboden und medialer Orbitawand (sog. „Transition Zone“ oder „Internal orbital buttresses“) bedeutsam.

- Eine Vergrößerung des Orbitavolumens durch eine trapezförmige knöcherne Resektion der lateralen Orbitawand in Richtung der Temporalgrube lässt sich nicht dauerhaft aufrechterhalten, ohne dass zusätzlich Implantate mit Platzhalterfunktion eingebracht werden [10].

Die große Bandbreite chirurgischer Alternativverfahren stützt sich zumeist auf invasivere Methoden aufseiten des operativen Zugangswegs ( $\mathrm{z}$. B. hemikoronarer Zugang) und auf das Ausmaß der chirurgischen Dekompressionsmaßnahmen (z.B. Komplettresektion des lateralen Orbitarands inklusive der lateralen Orbitawand).

Das hier vorgestellte Vorgehen von der Planung bis hin zur postoperativen Qualitätssicherung stellt zusammenfassend den Erfolg von Computerassistenz dar: Lediglich über einen inferioren transkonjunktivalen, retroseptalen Zugang werden sowohl die gezielte knöcherne Orbitawandresektion (z.B. von 3 Wänden) und auch die patientenspezifische Rekonstruktion vorhersagbar durchgeführt (Abb. 16).

\section{Fazit für die Praxis}

Die Basis für die virtuelle Operationsplanung und deren Nutzen für die Tumorchirurgie des Kopf-Hals-Bereichs stellt eine potente Bildanalyseplattform dar, die Bestandteil eines interaktiven Workflows sein muss, d.h., sie muss sowohl für die präoperative Analyse als auch für die intraoperative, interaktive Bildinfor- mationsausschöpfung (Navigation, intraoperatives CT/DVT) sowie für eine Anbindung an Schnittstellen, wie z.B. das postoperative Tumor-Staging oder eine Bestrahlungsplanung, geeignet sein. In Kombination mit der intraoperativen Navigation erleichtert dies die Orientierung und Resektion von Tumoren des Kopf-Hals-Bereichs mit besonderem Augenmerk auf die schädelbasisnahen Tumoren. Intraoperative Befunde und Daten zur Randschnittdiagnostik und Tumorausdehnung können digital gespeichert werden und bedienen die Schnittstellen zur Pathologie, Strahlentherapie und Radiologie. Des Weiteren erlaubt die computerassistierte präoperative Planung die Optimierung der Rekonstruktion durch die Herstellung patientenspezifischer Implantate - idealerweise in Kombination mit der Navigation zu deren Lagekontrolle. Durch die Verwendung patientenspezifischer Implantate, insbesondere im Bereich des Mittelgesichts, konnte im Laufe der letzten Jahre für viele Patienten ein Funktionserhalt von Bulbus und Augenhöhle gewährleistet werden, wo in Vorzeiten nur unzureichende Rekonstruktionen möglich waren bzw. - um Doppelbildproblematiken $\mathrm{zu}$ vermeiden - unter funktionellen Aspekten sogar exenteriert worden war. Insofern stellt die Implementierung patientenspezifischer Implantate in den Behandlungsablauf von Malignompatienten einen der Meilensteine chirurgischer Weiterentwicklungen aufgrund verbesserter Medizintechnik dar. Hier haben insbesondere 2 interaktive Bildanalyseplattformen der Medizinentwicklung bedeutsamen Vorschub geleistet: hierbei handelt es sich um die Plattformen der Firma BrainLAB (iPlan, München) und IVS-Solutions (VoXim, Chemnitz).

Der Hauptvorteil der virtuellen prä-, intra- und postoperativen virtuellen Planung ist im Sinne einer Qualitätssicherung und Kontrolle der Behandlungsschritte zu sehen. Die Bildfusion hat aufgrund der Supimposition verschiedener Bildmodalitäten mit unterschiedlicher Aussagekraft, aber auch bei der objektiven Verlaufskontrolle während einer Tumortherapie (z.B. Ansprechen auf Radio- und/oder Chemotherapie) eine große Bedeutung. Der Bereich der onkologischen Chirurgie ist ein Paradebeispiel für Schnittstellenoptimierung auf der Basis einer sinnvoll eingesetzten Computerassistenz, und zwar sowohl innerhalb des
Behandlungsablaufs eines individuellen Patienten als auch für die ebenso wichtige interdisziplinäre Kommunikation.

\section{Literatur}

${ }^{1}$ Bell RB. Computer planning and intraoperative navigation in cranio-maxillofacial surgery. Oral Maxillofac Surg Clin North Am 2010; 22: 135-156

2 Hohlweg-Majert B et al. Navigational maxillofacial surgery using virtual models. World J Surg 2005; 29: 1530-1538

${ }^{3}$ Kokemueller $\mathrm{H}$ et al. Complex midfacial reconstruction: a combined technique of computer-assisted surgery and microvascular tissue transfer. J Oral Maxillofac Surg 2008; 66: 2398-2406

${ }^{4}$ Schramm A et al. Computer-assisted navigation in craniomaxillofacial tumors. J Craniofac Surg 2008; 19: 1067-1074

${ }^{5}$ Schramm A, et al. Computer-assisted therapy in orbital and mid-facial reconstructions. Int J Med Robot 2009; 5: 111-124

${ }^{6}$ Stuehmer $C$ et al. Intraoperative navigation assisted reconstruction of a maxillo-facial gunshot wound. Oral Maxillofac Surg 2008; 12: 199-203

7 Gellrich NC et al. Computer-assisted secondary reconstruction of unilateral posttraumatic orbital deformity. Plast Reconstr Surg 2002; 110: 1417-1429

${ }^{8}$ Gellrich $N C$ et al. The lateral upper arm free flap for intraoral reconstruction. Int J Oral Maxillofac Surg 2000; 29: 104-111

${ }^{9}$ Gellrich NC et al. Follow-up in patients with oral cancer. J Oral Maxillofac Surg 2002; 60: 380-386

${ }^{10}$ Tavassol F et al. A quantitative approach to orbital decompression in Graves' disease using computer-assisted surgery: a compilation of different techniques and introduction of the "temporal cage". J Oral Maxillofac Surg 2011 [Epub ahead of print 15 July]

Dr. med. Dr. med. dent. Harald Essig Facharzt

Prof. Dr. med. Dr. med. dent. Martin Rücker

Leitender Oberarzt - stellvertretender ärztlicher Direktor

Dr. med. Dr. med. dent.

Frank Tavassol

Oberarzt

Dr. med. Dr. med. dent.

Horst Kokemüller

Geschäftsführender Oberarzt

Prof. Dr. med. Dr. med. dent. Nils-Claudius Gellrich

Ärztlicher Direktor

Klinik und Poliklinik für Mund-,

Kiefer- und Gesichtschirurgie

Zentrum Zahn-, Mund- und

Kieferheilkunde

Medizinische Hochschule Hannover

Carl-Neubergs-Straße 1

30625 Hannover

h.essig@gmail.com 